\title{
Faithful analytical effective one body waveform model for spin-aligned, moderately eccentric, coalescing black hole binaries
}

\author{
Danilo Chiaramello ${ }^{1,2}$ and Alessandro Nagar ${ }^{2,3}$ \\ 1 Dipartimento di Fisica, Università di Torino, via P. Giuria 1, 10125 Torino, Italy \\ ${ }^{2}$ INFN Sezione di Torino, Via P. Giuria 1, 10125 Torino, Italy and \\ ${ }^{3}$ Institut des Hautes Etudes Scientifiques, 91440 Bures-sur-Yvette, France
}

(Dated: October 29, 2020)

\begin{abstract}
We present a new effective-one-body (EOB) model for eccentric binary coalescences. The model stems from the state-of-the-art model TEOBiResumS_SM for circularized coalescing black-hole binaries, that is modified to explicitly incorporate eccentricity effects both in the radiation reaction and in the waveform. Using Regge-Wheeler-Zerilli type calculations of the gravitational wave losses as benchmarks, we find that a rather accurate $(\sim 1 \%)$ expression for the radiation reaction along mildly eccentric orbits $(e \sim 0.3)$ is given by dressing the current, EOB-resummed, circularized angular momentum flux, with a leading-order (Newtonian-like) prefactor valid along general orbits. An analogous approach is implemented for the waveform multipoles. The model is then completed by the usual merger-ringdown part informed by circularized numerical relativity (NR) simulations. The model is validated against the 22, publicly available, NR simulations calculated by the Simulating eXtreme Spacetime (SXS) collaboration, with mild eccentricities, mass ratios between 1 and 3 and up to rather large dimensionless spin values $( \pm 0.7)$. The maximum EOB/NR unfaithfulness, calculated with Advanced LIGO noise, is at most of order 3\%. The analytical framework presented here should be seen as a promising starting point for developing highly-faithful waveform templates driven by eccentric dynamics for present, and possibly future, gravitational wave detectors.
\end{abstract}

\section{INTRODUCTION}

Parameter estimates of all gravitational wave (GW) signals from coalescing binaries are done under the assumption that the inspiral is quasi-circular [1. This is motivated by the efficient circularization of the inspiral due to gravitational wave emission. In addition, no explicit evidence for eccentricity for some events was found 24 . However, recent population synthesis studies 5 -8] suggest that active galactic nuclei and globular clusters may host a population of eccentric binaries. Currently, there are no ready-to-use waveform models that accurately combine both eccentricity and spin effects over the entire parameter space. Recently, numerical relativity (NR) started producing surveys of eccentric, spinning binary black hole $(\mathrm{BBH})$ coalescence waveforms 9 11], and a NR-surrogate waveform model for nonspinning eccentric binaries up to mass ratio $q=10$ exists 11. On the analytical side, Refs. [12, 13 provided closed-form eccentric inspiral templates (based on the Quasi-Keplerian approximation). Similarly, a few exploratory effectiveone-body (EOB)-based [14 17] studies were recently performed [18 20]. In particular Refs. [18, 20] introduced and tested SEOBNRE, a way to incorporate eccentricity within the SEOBNRv1 21 circularized waveform model. However, the SEOBNRv1 model is outdated now, since it does not accurately cover high-spins, nor mass ratios up to 10 . This drawback is inherited by the SEOBNRE model [20].

In this article, we modify a highly NR-faithful EOB multipolar waveform model for circularized coalescing BBHs, TEOBiResums_SM [22, 23, to incorporate eccentricity-dependent effects. The EOB formalism relies on three building blocks: (i) a Hamiltonian, that describes the conservative part of the relative dynamics; (ii) a radiation reaction force, that accounts for the back-reaction onto the system due to the GW losses of energy and angular momentum; (iii) a prescription for computing the waveform. Including eccentricity requires modifications to blocks (ii) and (iii) with respect to the quasi-circular case.

\section{RADIATION REACTION AND WAVEFORM FOR ECCENTRIC INSPIRALS}

Within the EOB formalism, we use phase-space variables $\left(r, \varphi, p_{\varphi}, p_{r_{*}}\right)$, related to the physical ones by $r=$ $R /(G M)$ (relative separation), $p_{r_{*}}=P_{R_{*}} / \mu$ (radial momentum), $p_{\varphi}=P_{\varphi} /(\mu G M)$ (angular momentum) and $t=T /(G M)$ (time), where $\mu \equiv m_{1} m_{2} / M$ and $M=m_{1}+$ $m_{2}$. The radial momentum is $p_{r_{*}} \equiv(A / B)^{1 / 2} p_{r}$, where $A$ and $B$ are the EOB potentials. The EOB Hamiltonian is $\hat{H}_{\mathrm{EOB}} \equiv H_{\mathrm{EOB}} / \mu=\nu^{-1} \sqrt{1+2 \nu\left(\hat{H}_{\mathrm{eff}}-1\right)}$, with $\nu \equiv \mu / M$ and $\hat{H}_{\text {eff }}=\tilde{G} p_{\varphi}+\hat{H}_{\text {eff }}^{\text {orb }}$, where $\tilde{G} p_{\varphi}$ incorporates odd-in-spin (spin-orbit) effects while $\hat{H}_{\text {eff }}^{\text {orf }}$ incorporates even-in-spin effects [22]. We denote dimensionless spin variables as $\chi_{i} \equiv S_{i} / m_{i}^{2}$. The TEOBiResumS_SM [2325. waveform model is currently the most NR faithful model versus the zero_det_highP Advanced LIGO design sensitivity 26]). Reference 23 found that the maximum value of the EOB/NR unfaithfulness is always below $0.5 \%$ all 27 over the current release of the SXS NR waveform catalog 28 40. This is achieved by NR informing a $4.5 \mathrm{PN}$ spin-orbit effective function $c_{3}\left(\nu, \chi_{1}, \chi_{2}\right)$ and an effective 5PN function $a_{6}^{c}(\nu)$ entering the Padé resummed radial potential $A(r)$. [see Eqs. (39) and (33) of 

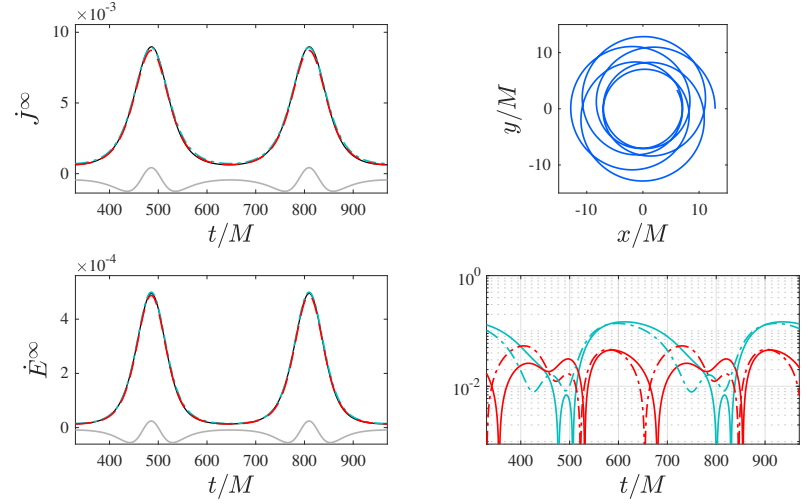

FIG. 1. Test-particle orbiting a Schwarzschild black hole with semilatus rectum $p=9$ and eccentricity $e=0.3$. Different analytical representations of the angular momentum and energy fluxes $\left(\dot{J}^{\infty}, \dot{E}^{\infty}\right)$ are compared with the numerical ones using the Regge-Wheeler-Zerilli (RWZ) formalism (black). Left panels: the nonresummed 2PN-accurate ones of Ref. 44, (gray); the resummed version via Eq. (5) (light-blue); the resummed version using the noncircular Newtonian prefactor (red), Eq. 8). The relative differences in $\left(\dot{J}^{\infty}, \dot{E}^{\infty}\right)$ are shown in the bottom-right panel: $\left|\delta \dot{J}^{\infty} / \dot{J}_{\mathrm{RWZ}}^{\infty}\right|$ (solid) and $\left|\delta \dot{E}^{\infty} / \dot{E}_{\mathrm{RWZ}}^{\infty}\right|$ (dashed), color scheme as above. On average, Eq. 8) delivers the closest analytical/numerical agreement.

Ref. [41]. The two Hamilton's equations that take account of GW losses are

$$
\begin{aligned}
\dot{p}_{\varphi} & =\hat{\mathcal{F}}_{\varphi}, \\
\dot{p}_{r_{*}} & =\sqrt{\frac{A}{B}}\left(-\partial_{r} \hat{H}_{\mathrm{EOB}}+\hat{\mathcal{F}}_{r}\right),
\end{aligned}
$$

where $\left(\hat{\mathcal{F}}_{\varphi}, \hat{\mathcal{F}}_{r}\right)$ are the two radiation reaction forces. In the quasi-circular case [22, 42] one sets $\hat{\mathcal{F}}_{r}=0$. Here, we use $\hat{\mathcal{F}}_{r} \neq 0$ and $\hat{\mathcal{F}}_{\varphi}$ explicitly includes noncircular terms. The main technical issue is to build (resummed) expressions of $\left(\hat{\mathcal{F}}_{\varphi}, \hat{\mathcal{F}}_{r}\right)$ that are reliable and robust up to merger. Building upon Ref. 43, Ref. 44 derived the $2 \mathrm{PN}$-accurate, generic expressions of $\left(\hat{\mathcal{F}}_{\varphi}, \hat{\mathcal{F}}_{r}\right)$, which are unsuited to drive the transition from the EOB inspiral to plunge and merger: they are nonresummed and generally unreliable in the strong-field regime (see below). The forces are related to the instantaneous losses of energy and angular momentum through GWs. Following Ref. 44, there exists a gauge choice such that the balance equations read

$$
\begin{aligned}
-\dot{J}^{\infty} & =\mathcal{F}_{\varphi}, \\
-\dot{E}^{\infty} & =\dot{r} \mathcal{F}_{r}+\dot{\varphi} \mathcal{F}_{\varphi}+\dot{E}_{\text {Schott }},
\end{aligned}
$$

where $E_{\text {Schott }}$ is the Schott energy (see Ref. [44, 45] and references therein), $(\dot{E}, \dot{J})^{\infty}$ are the energy and angular momentum fluxes at infinity, while $\mathcal{F}_{\varphi, r} \equiv \mu \hat{\mathcal{F}}_{\varphi, r}$. To build the resummed expressions of the functions $\left(\mathcal{F}_{\varphi}, \mathcal{F}_{r}, \dot{E}_{\text {Schott }}\right)$ and evaluate their strong-field reliability, we adopt the procedure that proved fruitful in the circularized case [24, 25, 46-49]: any analytical choice for $\left(\mathcal{F}_{\varphi}, \mathcal{F}_{r}, \dot{E}_{\text {Schott }}\right)$ is tested by comparisons with the energy and angular momentum fluxes emitted by a test particle orbiting a Schwarzschild black hole on eccentric orbits. We focus first on $\mathcal{F}_{\varphi}$. We start with the 2PN-accurate result of Ref. 44] [see Eq. (3.70) and Appendix D therein], $\mathcal{F}_{\varphi}^{2 \mathrm{PN}}\left(r, p_{r}, p_{\varphi}\right)$, reexpress it in terms of $p_{r_{*}}$, and factor it in a circular part (defined imposing $\left.p_{r_{*}}=\dot{p}_{r_{*}}=0\right), \mathcal{F}_{\varphi}^{2 \mathrm{PN}_{\mathrm{c}}}(r)$, and a noncircular contribution, $\mathcal{F}_{\varphi}^{2 \mathrm{PN}_{\mathrm{nc}}}\left(r, p_{r_{*}}, p_{\varphi}\right)$, so that $\mathcal{F}_{\varphi}^{2 \mathrm{PN}}\left(r, p_{r_{*}}, p_{\varphi}\right)=$ $\mathcal{F}_{\varphi}^{2 \mathrm{PN}_{\mathrm{c}}}(r) \mathcal{F}_{\varphi}^{2 \mathrm{PN}_{\mathrm{nc}}}\left(r, p_{r_{*}}, p_{\varphi}\right)$. A route to improve the strong-field behavior of this expression is to replace $\mathcal{F}_{\varphi}^{2 \mathrm{PN}_{\mathrm{c}}}(r)$ with the corresponding EOB-resummed expression [47] (notably, in its latest avatar [23 25]). To do so, the radial EOB coordinate $r$ in $\mathcal{F}_{\varphi}^{2 \mathrm{PN}_{\mathrm{c}}}(r)$ is first replaced by the circularized frequency variable $x \equiv \Omega_{\text {circ }}^{2 / 3}$, Eq. (5.22) of Ref. [4] at $2 \mathrm{PN}$ accuracy; then this $2 \mathrm{PN}$-accurate expression is replaced by $\mathcal{F}_{\varphi}^{\mathrm{EOB}_{\mathrm{c}}}(x)=$ $-32 / 5 \nu^{2} x^{7 / 2} \hat{f}(x)$, where $\hat{f} \equiv\left(F_{22}^{\text {Newt }}\right)^{-1} \sum_{\ell m} F_{\ell m}$ is the factored flux function [47, with all multipoles (except $m=0$ ones) up to $\ell=8$. Finally, the function $\mathcal{F}_{\varphi}^{\mathrm{EOB}} \mathrm{c}(x)$ is computed along the noncircular dynamics. We do so by using the circular frequency $\Omega_{\text {circ }} \equiv$ $\left.\partial_{p_{\varphi}} \hat{H}_{\mathrm{EOB}}\right|_{p_{\varphi}=j, p_{r_{*}}=0}$, where $j^{2} \equiv-A^{\prime}(u) /\left(u^{2} A(u)\right)^{\prime}$ is the (squared) circular angular momentum, $u \equiv r^{-1}$ and $(\cdot)^{\prime} \equiv \partial_{u}$. Note that in the resummed flux, we use $\left\{p_{\varphi}, \hat{H}_{\mathrm{EOB}}\left(r, p_{r_{*}}, p_{\varphi}\right), \hat{H}_{\mathrm{eff}}\left(r, p_{r_{*}}, p_{\varphi}\right)\right\}$ computed along the general dynamics. The $2 \mathrm{PN}$-accurate noncircular contribution $\mathcal{F}_{\varphi}^{2 \mathrm{PN}_{\mathrm{nc}}} \equiv f_{\varphi}^{N_{\mathrm{nc}}}+c^{-2} f_{\varphi}^{1 \mathrm{PN}_{\mathrm{nc}}}+c^{-4} f_{\varphi}^{2 \mathrm{PN}_{\mathrm{nc}}}$ is resummed using a $(0,2)$ Padé approximant. We have

$$
\mathcal{F}_{\varphi}^{\mathrm{EOB}_{2 \mathrm{PN}_{\mathrm{nc}}}} \equiv \mathcal{F}_{\varphi}^{\mathrm{EOB}_{\mathrm{c}}}(x(r)) P_{2}^{0}\left[\mathcal{F}_{\varphi}^{2 \mathrm{PN}_{\mathrm{nc}}}\left(r, p_{r_{*}}, p_{\varphi}\right)\right] .
$$

Alternatively, we recall that the force used to drive the EOB quasi-circular inspiral is

$$
\mathcal{F}_{\varphi}^{\mathrm{EOB}_{\mathrm{qc}}}=-\frac{32}{5} \nu^{2} r_{\omega}^{4} \Omega^{5} \hat{f}(\Omega)
$$

where $\Omega \equiv \dot{\varphi}$, which yields a more faithful representation of GW losses during the plunge [50, 51]. This expression is the leading quasi-circular term of the Newtonian angular momentum flux, obtained from Eq. (3.26) of Ref. [4, neglecting higher-order derivatives of $(r, \Omega)$. We can thus improve Eq. (6) multiplying it with the Newtonian noncircular factor

$$
\begin{aligned}
& \hat{f}_{\varphi}^{\text {Newt }}{ }^{\text {nc }}=1+\frac{3}{4} \frac{\ddot{r}^{2}}{r^{2} \Omega^{4}}-\frac{\ddot{\Omega}}{4 \Omega^{3}}+\frac{3 \dot{r} \dot{\Omega}}{r \Omega^{3}} \\
& +\frac{4 \dot{r}^{2}}{r^{2} \Omega^{2}}+\frac{\ddot{\Omega} \dot{r}^{2}}{8 r^{2} \Omega^{5}}+\frac{3}{4} \frac{\dot{r}^{3} \dot{\Omega}}{r^{3} \Omega^{5}}+\frac{3}{4} \frac{\dot{r}^{4}}{r^{4} \Omega^{4}}+\frac{3}{4} \frac{\dot{\Omega}^{2}}{\Omega^{4}} \\
& -\dddot{r}\left(\frac{\dot{r}}{2 r^{2} \Omega^{4}}+\frac{\dot{\Omega}}{8 r \Omega^{5}}\right)+\ddot{r}\left(-\frac{2}{r \Omega^{2}}+\frac{\ddot{\Omega}}{8 r \Omega^{5}}+\frac{3}{8} \frac{\dot{r} \dot{\Omega}}{r^{2} \Omega^{5}}\right)
\end{aligned}
$$

in order to get

$$
\mathcal{F}_{\varphi}^{\mathrm{EOB}_{\mathrm{Newt} \mathrm{nc}}}=-\frac{32}{5} \nu^{2} r_{\omega}^{4} \Omega^{5} \hat{f}_{\varphi}^{\mathrm{Newt} \mathrm{nc}_{\mathrm{nc}}} \hat{f}(\Omega)
$$



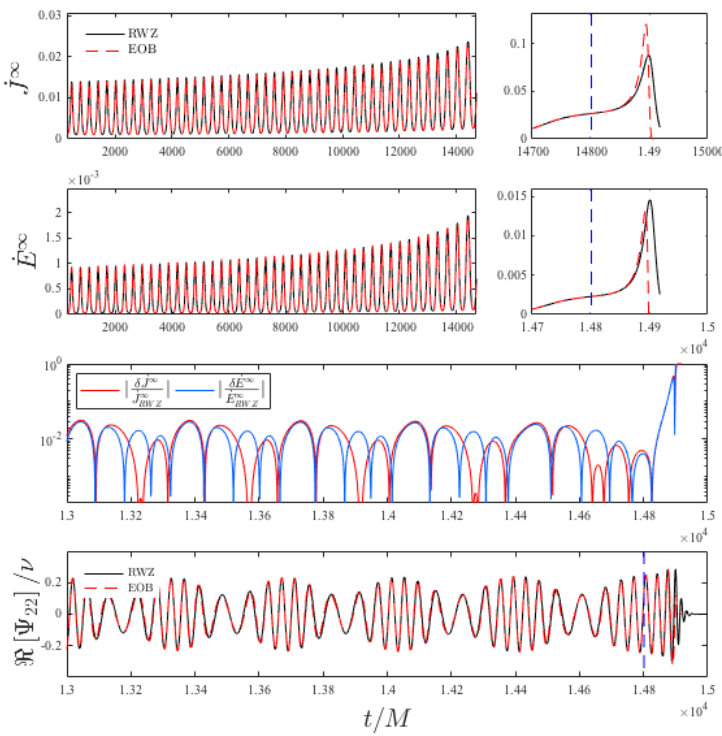

FIG. 2. Test particle (with mass ratio $\mu / M=10^{-3}$, $e=0.3$ ) and $p=8$ ) plunging over a Schwarzschild black hole. EOB/RWZ comparison between energy and angular momentum fluxes (top three panels) and waveforms (bottom panel). Vertical line: crossing of the stability threshold $(p=6+2 e)$ and beginning of the plunge.

Although this expression incorporates formally less noncircular PN information than Eq. (5), the timederivatives (and $\hat{f}(\Omega)$ as well) are obtained from the full EOB (resummed) equations of motion rather the $2 \mathrm{PN}$ ones used in $\mathcal{F}_{\varphi}^{2 \mathrm{PN}_{\mathrm{nc}}}$. For $\mathcal{F}_{r}$, we build on Ref. [44] and we use $\mathcal{F}_{r}=32 / 3 p_{r_{*}} / r^{4} P_{2}^{0}\left[\hat{\mathcal{F}}_{r}^{2 \mathrm{PN}}\right]$, where $P_{2}^{0}$ is the $(0,2)$ Padé approximant and $\hat{\mathcal{F}}_{r}^{2 \mathrm{PN}}=f_{r}^{N}+c^{-2} f_{r}^{1 \mathrm{PN}}+$ $c^{-4} f_{r}^{2 \mathrm{PN}}$ is the $2 \mathrm{PN}$ accurate expression calculated from Eqs. (3.70) and (D9-D11) of Ref. 44. We adopt an analogous approach to deal with the Schott energy, as given by Eqs. (3.57) and (C1-C4) of Ref. 44]. We factorize it in circular and noncircular parts that are both resummed with the $P_{2}^{0}$ Padé approximant, so to have $E_{\text {Schott }}=16 / 5 p_{r_{*}} / r^{3} P_{2}^{0}\left[E_{\text {Schott }}^{\mathrm{c}}\right] P_{2}^{0}\left[E_{\text {Schott }}^{\text {nc }}\right]$, where $E_{\text {Schott }}^{\text {nc }}=E_{\text {Schott }}^{\mathrm{nc}, 0}+c^{-2} E_{\text {Schott }}^{\mathrm{nc}, 1 \mathrm{PN}}+c^{-4} E_{\mathrm{Schott}}^{\mathrm{nc}, 2 \mathrm{PN}} . \quad$ Equations (5)-(8) are specialized to the test particle limit $(\nu=0)$ and computed along the eccentric, conservative, dynamics of a particle orbiting a Schwarzschild black hole. The result is compared with the fluxes computed using Regge-Wheeler-Zerilli (RWZ) black hole perturbation theory 52 54. To accurately extract waves at future null infinity, we adopt the hyperboloidal layer method of [55] and compute the fluxes with the usual expressions 56] of Ref. [54, including all multipoles up to $\ell=8$. Figure 1 shows the illustrative case of an orbit with semilatus rectum $p=9$ and eccentricity $e=0.3$. The apastron is $r_{1}=p /(1-e)$, and the periastron is $r_{2}=p /(1+e)$ [57. The figure indicates that $\mathcal{F}_{\varphi}^{\mathrm{EOB}_{\mathrm{Newt} \mathrm{nc}}}$
TABLE I. SXS simulations with eccentricity analyzed in this work. From left to right: the ID of the simulation; the mass ratio $q \equiv m_{1} / m_{2} \geq 1$; the individual spins $\left(\chi_{1}, \chi_{2}\right)$; the estimated NR eccentricity at first apastron $e_{\omega}^{\mathrm{NR}}$; the initial EOB eccentricity $e^{\mathrm{EOB}}$ and apastron frequency $\omega_{a}^{\mathrm{EOB}}$ which allow us to get a good EOB/NR frequency agreement.

\begin{tabular}{ccccc|ll|c}
\hline \hline SXS & $q$ & $\chi_{1}$ & $\chi_{2}$ & $e_{\omega}^{\mathrm{NR}}$ & $e^{\mathrm{EOB}}$ & $\omega_{a}^{\mathrm{EOB}}$ & $\max (\bar{F})[\%]$ \\
\hline \hline 1355 & 1 & 0 & 0 & 0.062 & 0.089 & 0.0280475 & 1.30 \\
1356 & 1 & 0 & 0 & 0.102 & 0.1503 & 0.019077 & 1.03 \\
1359 & 1 & 0 & 0 & 0.112 & 0.18 & 0.021495 & 1.22 \\
1357 & 1 & 0 & 0 & 0.114 & 0.1916 & 0.019617 & 1.20 \\
1361 & 1 & 0 & 0 & 0.160 & 0.23437 & 0.02104 & 1.56 \\
1360 & 1 & 0 & 0 & 0.161 & 0.2415 & 0.019635 & 1.52 \\
1362 & 1 & 0 & 0 & 0.217 & 0.30041 & 0.0192 & 0.89 \\
1364 & 2 & 0 & 0 & 0.049 & 0.0843 & 0.025241 & 0.86 \\
1365 & 2 & 0 & 0 & 0.067 & 0.11 & 0.023987 & 1.00 \\
1367 & 2 & 0 & 0 & 0.105 & 0.1494 & 0.026078 & 0.92 \\
1369 & 2 & 0 & 0 & 0.201 & 0.309 & 0.01755 & 1.38 \\
1371 & 3 & 0 & 0 & 0.063 & 0.0913 & 0.029058 & 0.57 \\
1372 & 3 & 0 & 0 & 0.107 & 0.149 & 0.026070 & 0.95 \\
1374 & 3 & 0 & 0 & 0.208 & 0.31405 & 0.016946 & 0.78 \\
\hline 89 & 1 & -0.5 & 0 & 0.047 & 0.071 & 0.0178279 & 0.96 \\
1136 & 1 & -0.75 & -0.75 & 0.078 & 0.121 & 0.02728 & 0.58 \\
321 & 1.22 & +0.33 & -0.44 & 0.048 & 0.076 & 0.02694 & 1.47 \\
322 & 1.22 & +0.33 & -0.44 & 0.063 & 0.0984 & 0.026895 & 1.18 \\
323 & 1.22 & +0.33 & -0.44 & 0.104 & 0.141 & 0.025965 & 1.57 \\
324 & 1.22 & +0.33 & -0.44 & 0.205 & 0.2915 & 0.019067 & 2.25 \\
1149 & 3 & +0.70 & +0.60 & 0.037 & 0.0617 & 0.0266802 & 3.16 \\
1169 & 3 & -0.70 & -0.60 & 0.036 & 0.049 & 0.024285 & 0.17 \\
\hline \hline
\end{tabular}

delivers analytical energy and angular momentum fluxes (red lines) that are, on average, in better agreement with the RWZ ones than those obtained from $\mathcal{F}_{\varphi}^{\mathrm{EOB}} \mathrm{BPN}_{\mathrm{nc}}$, which increase up to a $10 \%$ fractional difference at apastron. We adopt then $\mathcal{F}_{\varphi}^{\mathrm{EOB} \mathrm{New}_{\mathrm{nc}}}$ as analytical representation of the angular momentum flux along generic orbits. The maximal analytical/RWZ flux relative differences are $\sim 10^{-2}$. The robustness of this result is checked by considering several orbits with $p$ varying from just above the stability threshold $(p=6+2 e)$ up to $p=21$, and for each $p$ we consider $0 \leq e \leq 0.9$. We then compute the relative flux differences $(\delta \dot{\vec{E}}, \delta \dot{J})$ at periastron for each $(p, e)$. We find that, for each value of $p,(\delta \dot{E}, \delta \dot{J})$ are at most of the order of $10 \%$ for $e=0.9$. More interestingly if $e \lesssim 0.3$, the fractional differences do not exceed the $5 \%$ level. Let us consider now the waveform emitted from the transition from inspiral to plunge, merger and ringdown as driven by $\left(\mathcal{F}_{r}, \mathcal{F}_{\varphi}^{\mathrm{EOB}_{\mathrm{New}_{\mathrm{nc}}}}\right)$, focusing on a testparticle (of mass ratio $\mu / M=10^{-3}$ ) on a Schwarzschild background. To efficiently compute, along the relative dynamics, up to the third time-derivative of the phasespace variables entering Eq. (7), we suitably generalize the iterative analytical procedure used in Appendix A 

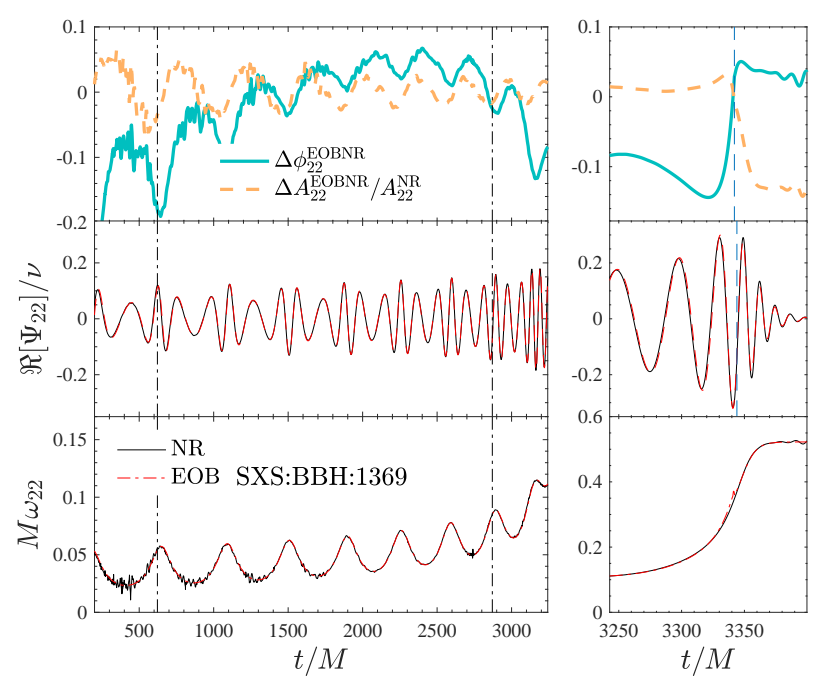

FIG. 3. Illustrative EOB/NR time-domain waveform comparison for the nonspinning configuration SXS : BBH : 1369, with $e_{\omega}^{\mathrm{NR}}=0.201, q=2$ and $\chi_{1}=\chi_{2}=0$.
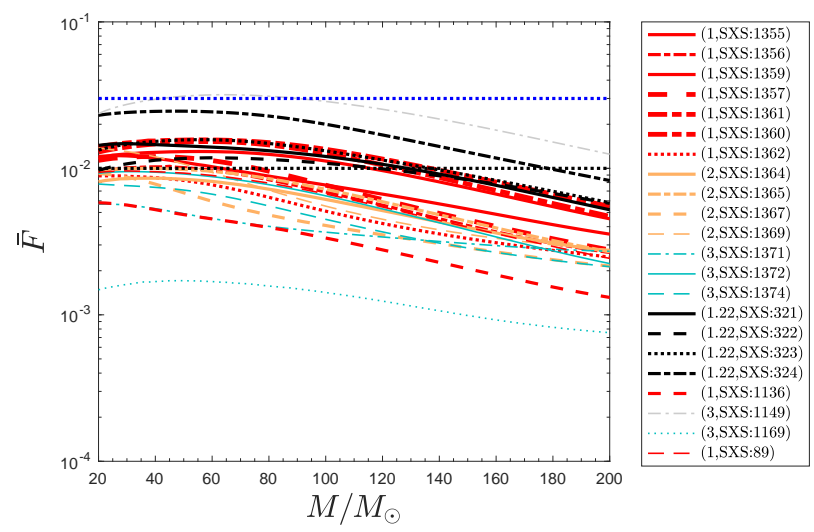

FIG. 4. EOB/NR unfaithfulness computed over the eccentric SXS simulations publicly available. The horizontal lines mark the $3 \%$ and $1 \%$ values.

of Ref. [58] to calculate $\ddot{r}$. We checked that two iterations are sufficient to obtain an excellent approximation $\left(\simeq 10^{-3}\right)$ of the derivatives computed numerically. An illustrative waveform is displayed in Fig. 2 for $p=8$ and $e=0.3$ (initial values). The top three rows of the figure highlight the numerical consistency $\left(\sim 10^{-2}\right)$ between the RWZ angular momentum and energy fluxes and their analytical counterparts. The corresponding waveform is shown (in black) in the fourth row of the plot. The gravitational waveform is decomposed in multipoles as $h_{+}-\mathrm{i} h_{\times}=D_{L}^{-1} \sum_{\ell m} h_{\ell m-2} Y_{\ell m}$, where $D_{L}$ is the luminosity distance and ${ }_{-2} Y_{\ell m}$ the $s=-2$ spin-weighted spherical harmonics. We use below the RWZ normalized variable $\Psi_{\ell m}=h_{\ell m} / \sqrt{(\ell+2)(\ell+1) \ell(\ell-1)}$. A detailed analysis of the properties of the RWZ waveform, such as the excitation of QNMs, etc., will be presented elsewhere. Here we employ it as a target, an "exact" waveform to validate the EOB one. Within the EOB for-

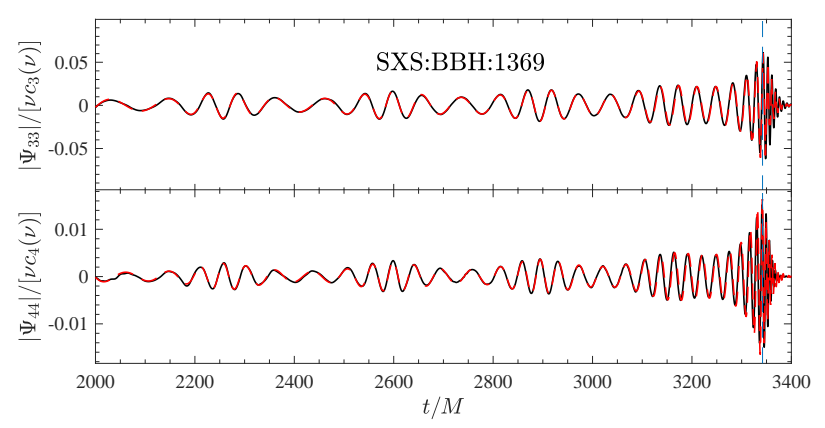

FIG. 5. Illustrative EOB/NR comparison for SXS:BBH: 1369 for the $(3,3)$ and $(4,4)$ waveform modes. Normalization constants are $c_{3}=\sqrt{1-4 \nu}$ and $c_{4}=1-3 \nu$.

malism, each multipole is factorized as $h_{\ell m}=h_{\ell m}^{(N, \epsilon)} \hat{h}_{\ell m}$, where $h_{\ell m}^{(N, \epsilon)}$ is the Newtonian (leading-order) prefactor, $\hat{h}_{\ell m}$ is the resummed relativistic correction [47] and $\epsilon$ the parity of $\ell+m$. The circularized prefactor $h_{\ell m}^{(N, \epsilon)}$ is replaced by its general expression obtained computing the time-derivatives of the Newtonian mass and current multipoles. We have $h_{\ell m}^{(N, 0)} \propto e^{\mathrm{i} m \varphi} I_{\ell m}^{(\ell)}$ and $h_{\ell m}^{(N, 1)} \propto$ $e^{\mathrm{i} m \varphi} S_{\ell m}^{(\ell)}$, where $(\ell)$ indicates the $\ell$-th time-derivative and $I_{\ell m} \equiv r^{\ell} e^{-\mathrm{i} m \varphi}$ and $S_{\ell m} \equiv r^{\ell+1} \Omega e^{-\mathrm{i} m \varphi}$ are the Newtonian mass and current multipoles. The $\ell=m=2$ mode of the analytical waveform is superposed, as a red line, in the bottom row of Fig. 2 showing excellent agreement with the RWZ one essentially up to merger [59]. A similar agreement is found for subdominant modes.

\section{COMPARISON WITH NUMERICAL RELATIVITY SIMULATIONS}

The complete, $\nu$-dependent, radiation reaction of above replaces now the standard one used in TEOBiResumS_SM, so to consistently drive an eccentric inspiral. Everything is analogous to the test-particle case, aside from (i) the initial conditions at the apastron, which are more involved because of the presence of spin, though they are a straightforward generalization of those of [19]; (ii) similar complications for the time-derivatives needed in $\hat{f}^{\mathrm{Newt}_{\mathrm{nc}}}$. The EOB waveform with the noncircular Newtonian prefactors is completed by next-to-quasi-circular (NQC) corrections and the NR-informed circularized ringdown [22, 23. Differently from the circularized case, the NQC correction factor is smoothly activated in time just when getting very close to merger, so to avoid spurious contaminations during the inspiral. Also, no iteration on the NQC amplitude parameters is performed [23. We assess the quality of the analytic waveforms by comparing them with the sample of eccentric NR simulations publicly available in the SXS catalog [28 40] that are listed in TableI. We carry out both time-domain comparisons and compute the EOB/NR unfaithfulness. To do so correctly 
the EOB evolution should be started in such a way that the eccentricity-induced frequency oscillations are consistent with the corresponding ones in the NR simulations. Since the eccentricities are gauge dependent, their nominal values are meaningless for this purpose. EOB and NR waveforms are then aligned in the time-domain [58] during the early inspiral and then we progressively vary the initial GW frequency at apastron, $\omega_{a}^{\mathrm{EOB}}$, and eccentricity, $e^{\mathrm{EOB}}$, until we achieve minimal fractional differences $\left(\simeq 10^{-2}\right)$ between the EOB and NR GW frequencies. To facilitate the parameter choice, we also estimate the initial (at first apastron) eccentricity of each NR simulation, $e_{\omega}^{\mathrm{NR}}$, using the method proposed in Eq. (2.8) of Ref. [10, where $e_{\omega}$ is deduced from the frequency oscillations; we here employ, however, the frequency of the $(2,2)$ mode, as opposed to the orbital frequency as done in Ref. 10]. The last two columns of Table I] contain the values of $\left(\omega_{a}^{\mathrm{EOB}}, e^{\mathrm{EOB}}\right)$ that lead to the best agreement between NR and EOB waveforms. An illustrative time-domain comparison, for SXS : BBH : 1369 is shown in Fig. 3. Figure 4 shows the EOB/NR unfaithfulness $\bar{F} \equiv 1-F$ (see Eq. (48) of Ref. 23]) computed with the zero_det_highP [26] Advanced-LIGO power spectral density. Both NR and EOB waveforms (starting at approximately the same frequency) were suitably tapered in the early inspiral. From Table I, $\max (\bar{F})$ is always comfortably below $3 \%$ except for the, small-eccentricity, dataset SXS:BBH: 1149 , with $\max (\bar{F})=3.16$. We believe that this is the effect of the suboptimal choice of $\left(a_{6}^{c}, c_{3}\right)$ (see below) and is not related to the modelization of eccentricity effects. By contrast, for large eccentricities, the $\bar{F}$ computation may be influenced by the accuracy of NR simulations, which get progressively more noisy increasing $e_{\omega}$ (see e.g. bottom panel of Fig. 3 , a similar behavior is also found for SXS:BBH:324). The accumulated phase difference at meger (always $\sim 1 \mathrm{rad}$ ) is mostly due to the previously determined [23] $\left(a_{6}^{c}, c_{3}\right)$ values, that depend on the circularized waveform and radiation reaction. Consistently, when our generalized framework is applied to circularized (nonspinning) binaries, we find that $\max (\bar{F})$ varies between $1.25 \%(q=1)$ and $0.21 \%$ $(q=8)$. These values are about one order of magni- tude larger than those of TEOBiResumS_SM(see Fig. 13 of [41]). Forthcoming work will present a retuning of $\left(a_{6}^{c}, c_{3}\right)$ so to improve the EOB/NR agreement further. Some subdominant multipoles are rather robust in the nonspinning case, see e.g. Fig. 5. For large spins, we find the same problems related to the correct determination of NQC corrections found for TEOBiResumS_SM [23]. Highly-accurate NR simulations covering a larger portion of the parameter space (see e.g. Ref. 10) are thus needed to robustly validate the model when $e_{\omega}^{\mathrm{NR}} \gtrsim 0.2$.

\section{CONCLUSIONS}

We illustrated that minimal modifications to TEOBiResumS_SM 23] enabled us to build a (mildly) eccentric waveform model that is reasonably NR-faithful over a nonnegligible portion of the parameter space. This model could provide new eccentricity measurements on LIGO-Virgo events. Our approach can be applied also in the presence of tidal effects. Higher-order corrections in the waveforms and flux (see Refs. [18, 19, 60] ) should be included to improve the model for larger eccentricities. In this respect, with a straighforward modification of the initial conditions 63, our model can also generate waveforms for dynamical captures or hyperbolic encounters [61, although NR validation is needed [62, 63. Provided high-order, gravitational-self-force informed, resummed expressions for the EOB potentials 64 68, as well as analytically improved fluxes to enhance the analytical/numerical agreement of Fig. 11 for larger eccentricities, we believe that our approach can pave the way to the efficient construction of EOB-based waveform templates for extreme mass ratio inspirals, as interesting sources for LISA [69, 70].

\section{ACKNOWLEDGMENTS}

We are grateful to T. Damour, G. Pratten and I. Romero-Shaw for useful comments, and to P. Rettegno for cross checking many calculations.
[1] B. P. Abbott et al. (LIGO Scientific, Virgo), Phys. Rev. X9, 031040 (2019), arXiv:1811.12907 [astro-ph.HE]

[2] B. P. Abbott et al. (Virgo, LIGO Scientific), Class. Quant. Grav. 34, 104002 (2017), arXiv:1611.07531 [grqc].

[3] A. H. Nitz, A. Lenon, and D. A. Brown, (2019), arXiv:1912.05464 [astro-ph.HE].

[4] I. M. Romero-Shaw, N. Farrow, S. Stevenson, E. Thrane, and X.-J. Zhu, (2020), arXiv:2001.06492 [astro-ph.HE]

[5] J. Samsing, M. MacLeod, and E. Ramirez-Ruiz, Astrophys. J. 784, 71 (2014), arXiv:1308.2964 [astro-ph.HE]

[6] C. L. Rodriguez, S. Chatterjee, and F. A. Rasio, Phys. Rev. D93, 084029 (2016), arXiv:1602.02444 [astro-
ph.HE]

[7] K. Belczynski, D. E. Holz, T. Bulik, and R. O'Shaughnessy, Nature 534, 512 (2016), arXiv:1602.04531 [astro-ph.HE],

[8] J. Samsing, $\quad$ Phys. Rev. D97, 103014 (2018), arXiv:1711.07452 [astro-ph.HE],

[9] I. Hinder, L. E. Kidder, and H. P. Pfeiffer, (2017), arXiv:1709.02007 [gr-qc].

[10] A. Ramos-Buades, S. Husa, G. Pratten, H. Estellés, C. García-Quirós, M. Mateu, M. Colleoni, and R. Jaume, (2019), arXiv:1909.11011 [gr-qc].

[11] E. A. Huerta et al., Phys. Rev. D100, 064003 (2019) arXiv:1901.07038 [gr-qc]. 
[12] A. Klein, Y. Boetzel, A. Gopakumar, P. Jetzer, and L. de Vittori, Phys. Rev. D98, 104043 (2018) arXiv:1801.08542 [gr-qc].

[13] S. Tiwari, G. Achamveedu, M. Haney, and P. Hemantakumar, Phys. Rev. D99, 124008 (2019) arXiv:1905.07956 [gr-qc].

[14] A. Buonanno and T. Damour, Phys. Rev. D59, 084006 (1999), arXiv:gr-qc/9811091

[15] A. Buonanno and T. Damour, Phys. Rev. D62, 064015 (2000), arXiv:gr-qc/0001013

[16] A. Buonanno, Y. Chen, and T. Damour, Phys. Rev. D74, 104005 (2006), arXiv:gr-qc/0508067.

[17] T. Damour, P. Jaranowski, and G. Schäfer, Phys. Rev. D91, 084024 (2015), arXiv:1502.07245 [gr-qc]

[18] Z. Cao and W.-B. Han, Phys. Rev. D96, 044028 (2017), arXiv:1708.00166 [gr-qc]

[19] T. Hinderer and S. Babak, Phys. Rev. D96, 104048 (2017), arXiv:1707.08426 [gr-qc]

[20] X. Liu, Z. Cao, and L. Shao, (2019), arXiv:1910.00784 $[\mathrm{gr}-\mathrm{qc}]$.

[21] A. Taracchini, Y. Pan, A. Buonanno, E. Barausse, M. Boyle, et al., Phys.Rev. D86, 024011 (2012) arXiv:1202.0790 [gr-qc]].

[22] A. Nagar et al., Phys. Rev. D98, 104052 (2018) arXiv:1806.01772 [gr-qc]

[23] A. Nagar, G. Riemenschneider, G. Pratten, P. Rettegno, and F. Messina, (2020), arXiv:2001.09082 [gr-qc]

[24] A. Nagar and A. Shah, Phys. Rev. D94, 104017 (2016) arXiv:1606.00207 [gr-qc]

[25] F. Messina, A. Maldarella, and A. Nagar, Phys. Rev. D97, 084016 (2018), arXiv:1801.02366 [gr-qc]

[26] D. Shoemaker, https://dcc.ligo.org/cgibin/DocDB/ShowDocument?docid=2974.

[27] Modulo a single outlier at $0.7 \%$.

[28] T. Chu, H. P. Pfeiffer, and M. A. Scheel, Phys. Rev. D80, 124051 (2009), arXiv:0909.1313 [gr-qc]

[29] G. Lovelace, M. Scheel, and B. Szilagyi, Phys.Rev. D83, 024010 (2011), arXiv:1010.2777 [gr-qc].

[30] G. Lovelace, M. Boyle, M. A. Scheel, and B. Szilagyi, Class. Quant. Grav. 29, 045003 (2012), arXiv:1110.2229 [gr-qc].

[31] L. T. Buchman, H. P. Pfeiffer, M. A. Scheel, and B. Szilagyi, Phys. Rev. D86, 084033 (2012), arXiv:1206.3015 [gr-qc].

[32] D. A. Hemberger, G. Lovelace, T. J. Loredo, L. E. Kidder, M. A. Scheel, B. Szilágyi, N. W. Taylor, and S. A. Teukolsky, Phys. Rev. D88, 064014 (2013), arXiv:1305.5991 [gr-qc]

[33] M. A. Scheel, M. Giesler, D. A. Hemberger, G. Lovelace, K. Kuper, M. Boyle, B. Szilágyi, and L. E. Kidder, Class. Quant. Grav. 32, 105009 (2015), arXiv:1412.1803 [gr-qc]

[34] J. Blackman, S. E. Field, C. R. Galley, B. Szilágyi, M. A. Scheel, M. Tiglio, and D. A. Hemberger, Phys. Rev. Lett. 115, 121102 (2015), arXiv:1502.07758 [gr-qc]

[35] G. Lovelace et al., Class. Quant. Grav. 32, 065007 (2015), arXiv:1411.7297 [gr-qc]

[36] A. H. Mroue, M. A. Scheel, B. Szilagyi, H. P. Pfeiffer, M. Boyle, et al., Phys.Rev.Lett. 111, 241104 (2013) arXiv:1304.6077 [gr-qc]

[37] P. Kumar, K. Barkett, S. Bhagwat, N. Afshari, D. A. Brown, G. Lovelace, M. A. Scheel, and B. Szilágyi, Phys. Rev. D92, 102001 (2015), arXiv:1507.00103 [gr-qc]

[38] T. Chu, H. Fong, P. Kumar, H. P. Pfeiffer, M. Boyle, D. A. Hemberger, L. E. Kidder, M. A. Scheel, and
B. Szilagyi, Class. Quant. Grav. 33, 165001 (2016) arXiv:1512.06800 [gr-qc].

[39] M. Boyle et al., Class. Quant. Grav. 36, 195006 (2019), arXiv:1904.04831 [gr-qc].

[40] "SXS Gravitational Waveform Database," https:// data.black-holes.org/waveforms/index.html.

[41] A. Nagar, G. Pratten, G. Riemenschneider, and R. Gamba, (2019), arXiv:1904.09550 [gr-qc].

[42] S. Ossokine et al., in preparation, 2019.

[43] A. Gopakumar, B. R. Iyer, and S. Iyer, Phys. Rev. D55, 6030 (1997), [Erratum: Phys. Rev.D57,6562(1998)], arXiv:gr-qc/9703075 [gr-qc]

[44] D. Bini and T. Damour, Phys.Rev. D86, 124012 (2012), arXiv:1210.2834 [gr-qc]

[45] T. Damour, A. Nagar, D. Pollney, and C. Reisswig, Phys.Rev.Lett. 108, 131101 (2012), arXiv:1110.2938 [grqc]

[46] T. Damour, B. R. Iyer, and B. S. Sathyaprakash, Phys. Rev. D57, 885 (1998), arXiv:gr-qc/9708034 [gr-qc]

[47] T. Damour, B. R. Iyer, and A. Nagar, Phys. Rev. D79, 064004 (2009), arXiv:0811.2069 [gr-qc]

[48] Y. Pan, A. Buonanno, R. Fujita, E. Racine, and H. Tagoshi, Phys.Rev. D83, 064003 (2011), arXiv:1006.0431 [gr-qc]

[49] A. Nagar, F. Messina, C. Kavanagh, G. LukesGerakopoulos, N. Warburton, S. Bernuzzi, and E. Harms, Phys. Rev. D100, 104056 (2019), arXiv:1907.12233 [gr-qc].

[50] T. Damour and A. Gopakumar, Phys. Rev. D73, 124006 (2006), arXiv:gr-qc/0602117.

[51] T. Damour and A. Nagar, Phys. Rev. D76, 064028 (2007), arXiv:0705.2519 [gr-qc]

[52] T. Regge and J. A. Wheeler, Phys. Rev. 108, 1063 (1957).

[53] F. J. Zerilli, Phys. Rev. Lett. 24, 737 (1970).

[54] A. Nagar and L. Rezzolla, Class. Quant. Grav. 22, R167 (2005), arXiv:gr-qc/0502064.

[55] S. Bernuzzi, A. Nagar, and A. Zenginoglu, Phys.Rev. D84, 084026 (2011), arXiv:1107.5402 [gr-qc]

[56] We removed the (negligible) $m=0$ modes since their analytic representation is poor.

[57] Semilatus rectum and eccentricity are defined by their relationships with the apastron and periastron radii. Explicit formulae relating $\left(E, p_{\varphi}\right)$ and $(p, e)$, which we use to set the initial data for an orbit specified by its eccentricity and semilatus rectum, as well as to compute $p$ and $e$ at each step of the motion, can be derived by solving the equations: $E=\hat{H}_{\mathrm{EOB}}\left(r_{1,2}, p_{\varphi}, p_{r_{*}}=0\right)$; finding, e.g., in the Schwarzschild case: $E^{2}=\left((p-2)^{2}-\right.$ $\left.4 e^{2}\right) /\left(p\left(p-3-e^{2}\right)\right)$ and $p_{\varphi}^{2}=p^{2} /\left(p-3-e^{2}\right)$.

[58] T. Damour, A. Nagar, and S. Bernuzzi, Phys.Rev. D87, 084035 (2013), arXiv:1212.4357 [gr-qc]

[59] The analytical waveform is not completed with any RWZinformed representation of merger and postmerger phase.

[60] C. K. Mishra, K. G. Arun, and B. R. Iyer, Phys. Rev. D91, 084040 (2015), arXiv:1501.07096 [gr-qc]

[61] W. E. East, S. T. McWilliams, J. Levin, and F. Pretorius, Phys. Rev. D87, 043004 (2013), arXiv:1212.0837 [gr-qc]

[62] R. Gold and B. Brügmann, Phys. Rev. D88, 064051 (2013), arXiv:1209.4085 [gr-qc]

[63] T. Damour, F. Guercilena, I. Hinder, S. Hopper, A. Nagar, et al., (2014), arXiv:1402.7307 [gr-qc] 
[64] S. Akcay, L. Barack, T. Damour, and N. Sago, Phys. Rev. D86, 104041 (2012), arXiv:1209.0964 [gr-qc]

[65] D. Bini and T. Damour, Phys.Rev. D89, 064063 (2014) arXiv:1312.2503 [gr-qc]

[66] S. Akcay and M. van de Meent, Phys. Rev. D93, 064063 (2016), arXiv:1512.03392 [gr-qc]

[67] A. Antonelli, M. van de Meent, A. Buonanno, J. Steinhoff, and J. Vines, Phys. Rev. D101, 024024 (2020) arXiv:1907.11597 [gr-qc]

[68] L. Barack, M. Colleoni, T. Damour, S. Isoyama, and N. Sago, Phys. Rev. D100, 124015 (2019)
arXiv:1909.06103 [gr-qc]

[69] S. Babak, J. R. Gair, and R. H. Cole, Proceedings, 524th WE-Heraeus-Seminar: Equations of Motion in Relativistic Gravity (EOM 2013): Bad Honnef, Germany, February 17-23, 2013, Fund. Theor. Phys. 179, 783 (2015) arXiv:1411.5253 [gr-qc]

[70] C. P. L. Berry, S. A. Hughes, C. F. Sopuerta, A. J. K. Chua, A. Heffernan, K. Holley-Bockelmann, D. P Mihaylov, M. C. Miller, and A. Sesana, (2019), arXiv:1903.03686 [astro-ph.HE]. 